\title{
Availability analysis of mechanical systems with condition-based maintenance using semi-Markov and evaluation of optimal condition monitoring interval
}

\author{
Girish Kumar $^{1}$ - Vipul Jain ${ }^{2}$ O. P. Gandhi ${ }^{3}$
}

Received: 17 April 2015/ Accepted: 8 June 2017/Published online: 19 June 2017

(c) The Author(s) 2017. This article is an open access publication

\begin{abstract}
Maintenance helps to extend equipment life by improving its condition and avoiding catastrophic failures. Appropriate model or mechanism is, thus, needed to quantify system availability vis-a-vis a given maintenance strategy, which will assist in decision-making for optimal utilization of maintenance resources. This paper deals with semi-Markov process (SMP) modeling for steady state availability analysis of mechanical systems that follow condition-based maintenance (CBM) and evaluation of optimal condition monitoring interval. The developed SMP model is solved using two-stage analytical approach for steady-state availability analysis of the system. Also, CBM interval is decided for maximizing system availability using Genetic Algorithm approach. The main contribution of the paper is in the form of a predictive tool for system availability that will help in deciding the optimum CBM policy. The proposed methodology is demonstrated for a centrifugal pump.
\end{abstract}

Girish Kumar

girish.kumar154@gmail.com

Vipul Jain

vipul.jain@vuw.ac.nz

O. P. Gandhi

opgandhi_iitd@yahoo.com

1 Department of Mechanical Engineering, Delhi Technological University, Bawana Road, Delhi 110042, India

2 School of Management, Victoria Business School, Victoria University of Wellington, Wellington, New Zealand

3 ITMMEC, Indian Institute of Technology Delhi, Hauz Khas, Delhi 110016, India
Keywords Condition based maintenance - Availability · Semi-Markov process $\cdot$ Degraded states $\cdot$ Mechanical repairable systems

\section{List of symbols}

$A_{\mathrm{s}} \quad$ System availability

$A_{\text {pump }} \quad$ Pump availability

$D_{i} \quad i$ th degraded state, $i \in\{1,2,3, \ldots, n\}$

$D_{x_{i}} \quad i$ th degraded state for CM metric $x_{i}$

$F_{\mathrm{r}} \quad$ Random failure state

$F_{i j}(t) \quad$ CDF of the time spent in state $i$ before moving to state $j,(i, j \in \Omega)$

$\bar{F}_{i j}(t) \quad$ Complement of the $F_{i j}(t)$

$F_{i} \quad \mathrm{CDF}$ for CM interval for state $i$, where $i \in\left\{D_{x_{1}}, D_{x_{2}}, \ldots, D_{x_{n}}\right\}$

$\bar{F}_{i} \quad$ Complement of the CDF for CM interval for state $i$, where $i \in\left\{D_{x_{1}}, D_{x_{2}}, \ldots, D_{x_{n}}\right\}$

$\operatorname{im}_{p}^{r} \quad$ Imperfect repair state where repair carried out to $p$ th degraded state leads to $r$ th better degraded state, $p \in\{3, \ldots, n\}, r=1,2,3, \ldots, \mathrm{p}-2$

$K(t) \quad$ SMP Kernel matrix

$k_{i j}(t) \quad$ Element of the kernel matrix, $K(t)$ in $i$ th row and $j$ th column, $i, j \in \Omega$

$l_{i} \quad$ Lower bound of CM metric for $i$ th degraded state

$\mathrm{ma}_{\mathrm{k}} \quad$ Major repair state, where repair carried out at $k$ th degraded state and leads to first degraded state, $D_{1}, k \in\{2,3, \ldots, n\}$

$m i_{j} \quad$ Minimal repair state corresponding at $j$ th degraded state and maintenance leads to current degraded state, where $j \in\{1,2,3, \ldots, n\}$

$n \quad$ Number of degradation states for the system

$N \quad$ Total number states in the SMP model of the system

$p_{i j} \quad(i, j)$ th element of one step transition probability matrix, $Z$ 
$p_{j} \quad$ Probability of transitioning to state $j$ from CM state and $j$ is set of states having corresponding degraded state or possible maintenance state after CM

$P_{j} \quad$ Steady state probability of the system being in working state $j, j \in \mathrm{W}$

$P_{i} \quad$ Steady state probability of system being in state $i$, $i \in \Omega$

$S \quad$ System

$T_{i} \quad$ CM interval for degraded state $D_{i}$, $i \in\{1,2,3, \ldots, n\}$

$t$ Time

$u(\mathrm{t}-\mathrm{L}) \quad$ Unit step function

$u_{i} \quad$ Upper bound of CM metric for $i$ th degraded state

$W \quad$ Set of working states

$Z \quad$ One step transitional probability matrix

$\tau_{i} \quad$ Mean sojourn time of the $i$ th state, $i \in \Omega$

$\Omega \quad$ State space in the SMP model of system

$\beta \quad$ Shape parameter of Weibull distribution

$\beta_{i j} \quad$ Shape parameter of Weibull distribution for the time duration in $i$ th state and related to state $i$ to state $j$ transition

$\theta_{i j} \quad$ Weibull distribution scale parameter for the time duration in $i$ th state and related to state $i$ to state $j$ transition

$\theta \quad$ Weibull distribution scale parameter

$\mu_{i j} \quad$ Location parameter, mean, of log-normal distribution for the time duration in $i$ th state and related to state $i$ to state $j$ transition

$\sigma_{i j} \quad$ Scale parameter, standard deviation, of lognormal distribution for the time duration in $i$ th state and related to state $i$ to state $j$ transition

$\lambda \quad$ Parameter of the Exponential distribution

$\lambda_{i} \quad$ Exponential distribution parameter of the CM time for state $i$, where $i \in\left\{C_{1}, C_{2}, \ldots, C_{n}\right\}$

$H \quad$ Steady state probability matrix of the EMC

$h_{i} \quad$ Steady state probability of $i$ th state of the EMC, $i \in$ $\Omega$

\section{Introduction}

Mechanical systems are employed in numerous applications, such as power plant, aviation and manufacturing. These demand higher availability for economic considerations. The analysis of availability guides practicing engineers in selecting an appropriate maintenance strategy and in improving performance of the system (Kumar et al. 2009).

In the past, researchers have focused towards development of time-based maintenance models (Crocker and Kumar 2000). Preventive maintenance (PM) is the scheduled maintenance that includes actions such as; adjustments, inspection, lubrication and replacement of components (Sim and Endrenyi 1988). Ahmad et al. (2011) proposed a maintenance decision model for PM application. Charles-Owaba et al. (2008) suggested scheduling of the PM based on opportunistic cost. Though PM does help in avoiding the equipment breakdowns, but it no way fulfills maintenance objectives by not utilizing optimum use of maintenance resources. The replacement interval of components and lube oils under PM is conservative, and this result in their replacement, with sufficient residual life still left. To take care of this concern, CBM policy came up. Under this; a planned maintenance action, is undertaken, i.e., not timely based but condition based. In CBM, condition-monitoring (CM) techniques such as vibration, acoustic emission, thermography and oil and wear debris analysis are used to assess health of the system (Wang 2008).

Most of the mechanical systems experience gradual degradation. It is appropriate to consider multiple states of the considered mechanical system or component, instead of the binary states for availability analysis (Majid and Nasir 2011). Van et al. (2012) studied maintenance policy with stochastic degradation of equipment. In degradation models; the states are decided, in general, based on the realtime health of the system. Wang et al. (2010) proposed a multi-degrading states model and considered the periodic inspection at each degraded state with an appropriate maintenance action.

Condition-based maintenance modeling for mechanical systems has been reviewed by various researchers (Jardine et al. 2006; Pandian and Ali 2009) with noticeable contributions from Chen and Trivedi (2005); Carr and Wang (2011). However, the transition distributions for degradation, repair and inspection are considered exponential, which is not always applicable. Amari and McLaughlin (2004) and El-Damcese and Temraz (2011) demonstrated the analysis of a multi-state system using Markov reward model and Markov chain, respectively. Ahmad et al. (2017) suggested a simulation-based optimization approach for free distributed repairable multi-state availability-redundancy allocation problems. Semi-Markov process approach is, however, capable to handle non-exponential distributions. But these have been attempted for limited applications such as software (Xie et al. 2005), NC machine tool (Qiang et al. 2010) and opportunistic maintenance (Kumar et al. 2014).

Although extensive work has been carried out under CBM, yet little attention has been paid to availability modeling of systems with CBM maintenance (Khanduja et al. 2011). Moreover, the frequency of CM interval affects the system availability and the literature shows that there is limited work on optimization of CM interval. In this paper, SMP modeling of mechanical systems, with multi-state degradation and using CBM, is proposed for the steady-state availability analysis. 
The paper is organized, with system modeling described in the next Section. In Sect. 3, steady-state availability analysis is presented, while in Sect. 4, optimization of CM interval using GA is suggested and its methodology is described in Sect. 5. In Sect. 6, the proposed methodology is illustrated by an example and the last section, i.e., Sect. 7, concludes the paper.

\section{System modeling}

Condition-monitoring (CM) of the system is getting easier day-by-day, with recent advancements in sensor and realtime data capturing technologies. This has indeed helped to implement CBM in the plants in an effective way. In this work, a methodology for system availability assessment is developed, incorporating the CBM. The degradation behavior of the mechanical systems, together with periodic $\mathrm{CM}$ and their maintenance causes the stay time of their states that follow non-exponential distribution (Fricks and Trivedi 1997; Dennis 2003). The system model, therefore, is developed using the SMP, rather than a Markov model.

\section{Modeling degradation, random failures and condition monitoring}

In this subsection, three modeling aspects; degradation, random failures and periodic condition monitoring are considered. A SMP model of a mechanical system is developed employing the three features and is shown in Fig. 1.
Multi-degraded states with Weibull distribution are appropriate for developing the system model (Jager and Bertsche 2004). Let $D_{i}$, be a consecutive degraded state with $i \in\{1,2,3, \ldots, n\}$. Every degraded state, $D_{i}$, is identified using an appropriate CM metric, with lower and upper bounds; $l_{\mathrm{i}}$ and $u_{\mathrm{i}}$. It is assumed that there is gradual system degradation; with the degradation rate slow initially, which increases with time.

Application of the CBM for a system does rule out the occurrence of its random failure. But this has been considered in this work to take care of an unforeseen case, i.e., the system may fail randomly in spite of being under CM. The random failures are modeled with exponential distribution and may occur at any stage of degradation. A random failure in the model, Fig. 1, is shown by connecting the degraded state, $D_{i}$, to random failure state, $F_{r}$ by a directed line or edge, with direction from node $D_{i}$ to node $F_{r}$. It may be mentioned that the system is brought back to its first state, $D_{1}$, by an appropriate repair action and the lognormal distribution is considered for such repairs (Dennis 2003).

In principle, the CM interval between two consecutive $\mathrm{CM}$ checks should be longer in the initial degraded states, while it should be shorter in the subsequent and high degradation states. Therefore, CM intervals; $T_{1}, T_{2}, T_{3}, \ldots$, $T_{n}$, for the respective degraded states are considered in the decreasing order. Let $D_{x i}$, be the $i$ th degraded state for CM metric, $x_{i}$, with $l_{i} \leq x_{i} \leq u_{i}$. The system may iterate within the same degraded state, $D_{i}$, and within its metric range, by adopting or following the minimal repair. However, as the system ages, it moves to the next degraded state. The time

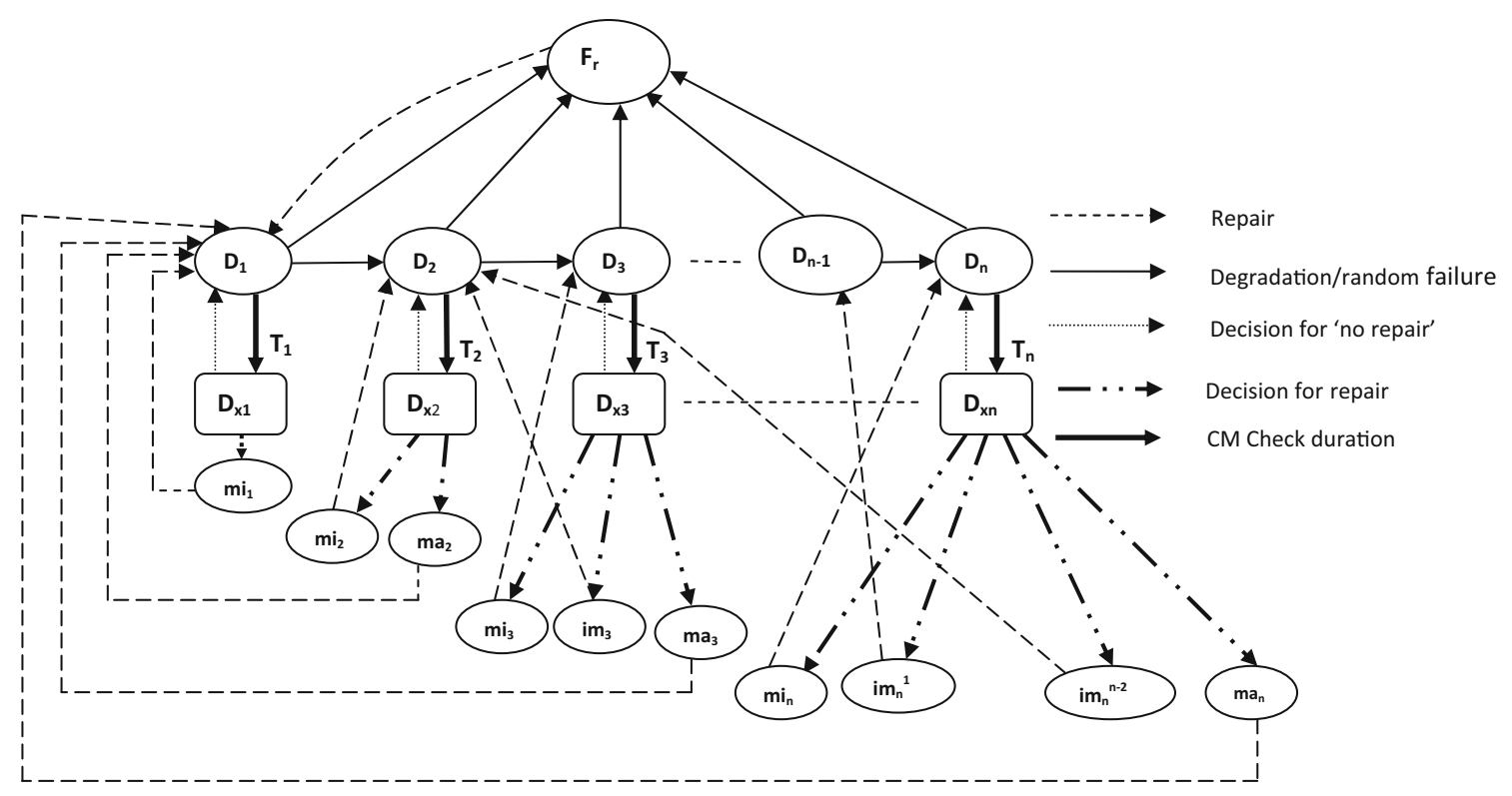

Fig. 1 System SMP model-CBM 
to perform CM at a degraded state is assumed to follow an exponential distribution.

\section{Modeling various repair actions}

The decision whether to undertake maintenance or maintain the status quo, is taken based on the condition of the system. In case the system health is normal and does not show deterioration from the $\mathrm{CM}$ metric check, the decision is 'no repair' and the system is allowed to operate. Otherwise, an appropriate maintenance action such as: minimal, imperfect or major repair is carried out to bring back the system to its current, previous better or the initial state, respectively.

In the system SMP model, Fig. 1, minimal repair is considered for all the degraded states, i.e., $\mathrm{mi}_{j}$,while major repair is considered for all degraded states except the first degraded state, i.e., $\operatorname{ma}_{k}, k \in\{2,3, \ldots, n\}$ and imperfect repair is considered at all degraded states, except the first and second degraded states, i.e., $\operatorname{im}_{p}^{r}, p \in$ $\{3, \ldots, n\}$ and $r=1,2,3, \ldots,(p-2)$. It is well known that a minimal repair takes, in general, the least time, while the imperfect repair takes more time than the minimal repair, but a lesser time than a major repair. Moreover, the time of repair will be higher, if the repair is carried out at a highly degraded state. The lognormal distribution, being more appropriate for repair of mechanical systems, is employed in the model (Dennis 2003).

The SMP system model, Fig. 1, is developed by incorporating the aspects discussed in this section. A solution procedure of the model for the system availability analysis is described in the next section.

\section{Steady-state availability analysis}

This section deals with steady state availability analysis based on the SMP system model in Sect. 2 and using analytical solution described in the following subsection.

\section{Analytical solution of the SMP model}

A two-stage method which is easy to implement, is selected for steady-state solution of the SMP model (Xie et al. 2005). In the two-stage method; Embedded Markov chain (EMC) of SMP that is a discrete time Markov chain (DTMC), is used to simplify the analysis of the SMP. Stage 1, deals with evaluation of the one-step transition probability matrix of Embedded Markov Chain of the SMP model, which is used to obtain steady-state probabilities of the EMC. In stage 2, the sojourn (stay) time of each state in a SMP model is evaluated and its steady-state probability is obtained using the values of the steady-state probabilities of the EMC (obtained from stage 1) and its sojourn time value. The procedure for both the stages is detailed in the following subsections.

\section{Stage 1: steady-state probabilities of EMC}

Refer Fig. 1 for the system SMP model, which consists of the state space, $\Omega$, represented as:

$$
\begin{aligned}
\Omega= & \left\{D_{1}, \ldots, D_{n} ; \quad F_{r} ; \quad D_{x_{1}}, \ldots, D_{x_{n}} ;\right. \\
& \left.m i_{1}, \ldots, m i_{n} ; \quad i m_{3}^{1}, \ldots, i m_{n}^{n-2} ; \quad m a_{2}, \ldots, m a_{n}\right\}
\end{aligned}
$$

The Semi-Markov Process model is expressed in terms of kernel matrix, $K(t)$, which is given as:

$$
\begin{aligned}
& \begin{array}{llllllllllllllllll}
D_{1} & \ldots & D_{n} & F_{r} & D_{x_{1}} & \ldots & D_{x_{n}} & m i_{1} & \ldots & m i_{n} & i m_{3}{ }^{1} & \ldots & i m_{n}{ }^{n-2} & m a_{2} & \ldots & m a_{n}
\end{array}
\end{aligned}
$$

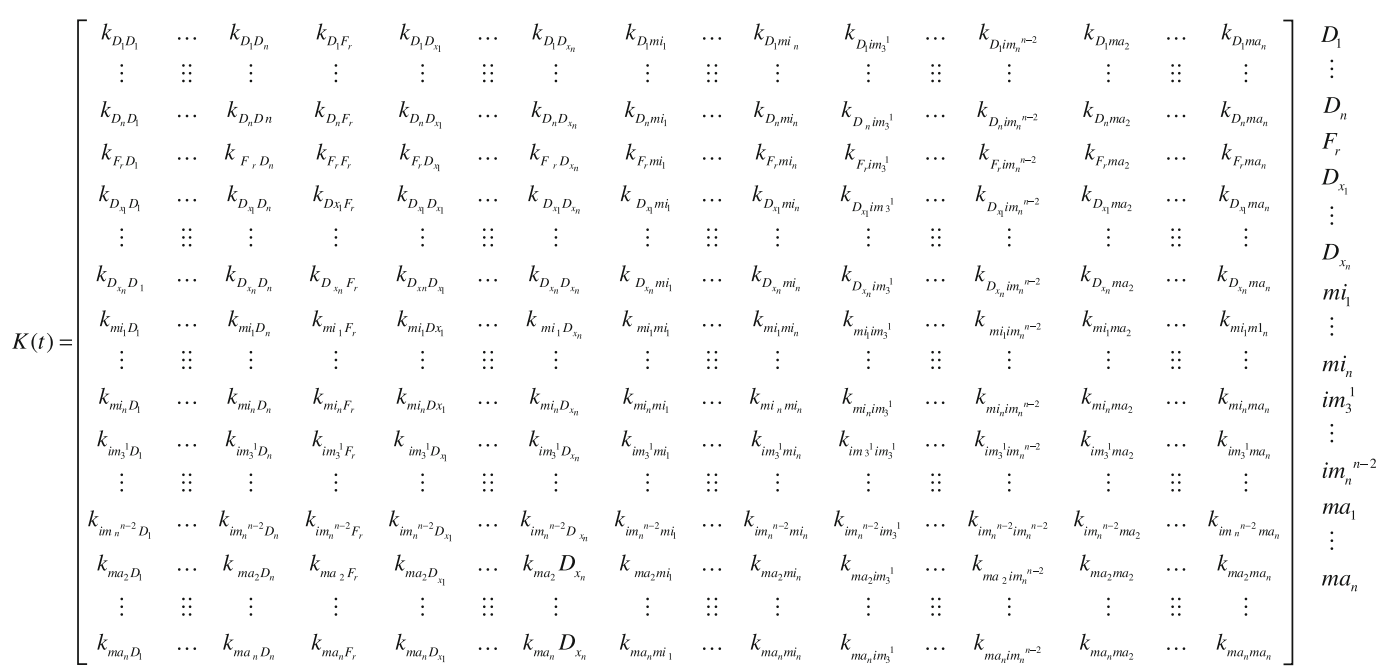


The elements, $k_{i j}(t)$ of the matrix, $K(t)$, as given by Kulkrani (2009) are redefined as:

$$
H=H K(\infty), \quad \sum_{i=1}^{N} h_{i}=1, i \in \Omega
$$

$k_{i j}(t)=\left\{\begin{array}{l}0 \\ F_{i j}(t) \\ \int_{0}^{t} \bar{F}_{i k} \bar{F}_{i m} \mathrm{~d} F_{i j}(x) \\ p_{j} F_{i}\end{array}\right.$
: when transition is not possible from the ith state within the time $t$

: when only one state $\mathrm{j}$ is reachable from the ith state (excluding the

CM states) within the time $t$

: when two or more states (for example; three states viz.j, kand $m$ )

are reachable from the ith state (excluding the CM state) within the

:when more than one state (i.e. degraded state and possible

maintenance states) reachable from the CM state $i, \mathrm{i} \in\left\{D_{x 1}, D_{x 2}, \ldots, D_{x n}\right\}$

within time $\mathrm{t}$
The Kernel matrix, $K(t)$, Eq. (2), is used to evaluate onestep transition probability matrix, $Z=K(\infty)$, of the EMC of the SMP by considering $t \rightarrow \infty$. Let the elements of the matrix, $Z$, be $p_{i j}$, with $i, j=1-N$. To evaluate these elements, there is an additional condition that for each row, the sum of elements of $K(t)$ becomes 1 , as $t \rightarrow \infty$, i.e., $\sum_{j=1}^{N} p_{i j}=1$ for all $i=1-N$. Since these elements involve complicated integrals for the chosen $\mathrm{CDF}$, these can be solved with the help of a software like Maple. The one-step transition probability matrix, $Z$, is given as (Kulkrani 2009):

$Z=K(\infty)=\left[\begin{array}{cccccc}p_{11} & p_{12} & p_{13} & \ldots & \ldots & p_{1 N} \\ p_{21} & p_{22} & p_{23} & \ldots & \ldots & p_{2 N} \\ p_{31} & p_{32} & p_{33} & \ldots & \ldots & p_{3 N} \\ \ldots & \ldots & \ldots & \ldots & \ldots & \ldots \\ \ldots & \ldots & \ldots & \ldots & \ldots & \ldots \\ p_{N 1} & p_{N 2} & p_{N 3} & \ldots & \ldots & p_{N N}\end{array}\right]$

The following system of linear equations is formulated to find the steady state probabilities of the EMC, $h_{i}, i \in \Omega$, (Kulkrani 2009). where $H=\left[h_{1}, h_{2}, \ldots, h_{N}\right]$ is the steady state probability matrix of the EMC and $K(\infty)$ is the Kernel matrix when $t \rightarrow \infty$.

The system of linear Equations, i.e., Eq. (4), can be solved by software such as Matlab. The steady state probabilities of the EMC, $h_{i}, i \in \Omega$, are obtained, and these values are used for evaluating steady state probability, $P_{i}, i \in \Omega$ of the SMP of state i, in stage 2 .

Stage 2: steady-state probabilities of states in SMP

In stage 2 , the mean sojourn time, $\tau_{i}$, i.e., the time the process spends at state $i$ is evaluated to obtain the steadystate probability of state $i$, i.e.; $P_{i}$. The mean sojourn time, $\tau_{i}$, is obtained using the following definition (Kulkrani 2009): $\tau_{i}=\left\{\begin{array}{l}0 \\ \int_{0}^{\infty} \bar{F}_{i j}(t) \mathrm{d} t \\ \int_{0}^{\infty} \bar{F}_{i j} \bar{F}_{i k} \bar{F}_{i m}(t) \mathrm{d} t \\ \int_{0}^{\infty} \bar{F}_{D_{x_{i}}} \mathrm{~d} t\end{array}\right.$
: when transition is not possible from the ith state within the time $t$

: when only one state $j$ is reachable from the ith state (excluding the CM states) within the time $t$

: when two or more states (for example; three states viz. $j, k$ and $m$ ) are reachable from the $i$ th state (excluding the CM state) within the time $t$ with three respective elements of

$k(t)$ in the $i$ th row

: when more than one state (i.e. degraded state and possible maintenance states) are reachable from the CM state $i$ $i \in\left\{D_{x 1}, D_{x 2}, \ldots, D_{x n}\right\}$ within time $t$ 
Using the values of steady state probabilities of the EMC and the sojourn time values, the steady state probability of state $i, P_{i}$, for the SMP model is obtained as (Kulkrani 2009):

$P_{i}=\frac{h_{i} \tau_{i}}{\sum_{j \in \Omega} h_{j} \tau_{j}}, \quad i \in \Omega$,

where $h_{i}$ and $\tau_{i}$ values are evaluated using Eqs. (4) and (5), respectively

If $\mathrm{W}$ is set of working/operating state, the steady state availability is given by:

$A_{s}=\sum_{j \in W} P_{j}$

where, $P_{j}$ is obtained using the Eq. (6).

\section{Optimization of CM interval using GA}

A genetic algorithm (GA) solves complex optimization problems and it is based on the natural evolution process (Goldberg 2006). In this approach, population changes continuously through cross breeding, mutation and natural selection. An explicit mathematical formulation is not required in implementation and, the parameter values, such as; population size, crossover rate and mutation rate are appropriately chosen to maintain the desired accuracy in the solution. The main steps of GA approach are (Kuo et al. 2001):

- Chromosome representation

- Generation of initial population

- Evaluation of fitness function

- Selection process

- Genetic operations (crossover, mutation)

- Selection of best chromosomes according to the fitness values

- Termination criteria

In this paper, the GA approach is applied to decide the optimal CM interval for maximizing the system availability. The availability function as per the procedure detailed in Sect. 3 is coded in Matlab and the developed code is used in the GA tool box for optimization.

A methodology based on the deliberations in Sects. 2, 3 and 4 is suggested for steady state system availability in the following section.

\section{Step wise methodology: availability analysis}

Steps for the steady-state availability analysis of the SMP model are presented below, which are based on the discussion in Sects. 2, 3 and 4.

\section{Step I: system structure}

Identify the components/subsystems in the selected system.

\section{Step II: SMP modeling of the system}

Derive feasible states for the system and evolve a SMP model (Refer Sect. 2).

\section{Step III: component/subsystem failure and repair distribution}

Identify the distribution and its parameter (s) for degradation/failure time, repair time, CM time interval and CM activity time at the component/subsystem level (Refer Sect. 2).

Step IV: two-stage steady state system availability analysis

\section{(a) Kernel matrix, $\mathrm{K}(\mathrm{t})$}

Derive the Kernel matrix, $K(t)$, for the system model using the definition of its elements, Eq. (2).

(b) One step transition probability matrix, Z, of the EMC

Generate one-step transition probability matrix, $Z$, of the EMC of the SMP model, using the step IV (a), with $t \rightarrow \infty$.

(c) Steady state probability of the states of EMC

Steady state probability of all states of EMC of the system SMP model, using Eq. (4).

(d) Mean sojourn time of the states

Evaluate mean sojourn time of all states of the SMP model, using Eq. (5).

(e) Steady state probability of states

Steady state probability of state $i, P_{i}$, of the SMP model is obtained using the Eq. (6).

\section{(f) Availability evaluation}

Using step IV (e), evaluate the system steady state availability by adding the steady-state probabilities of the working states in the system, i.e., Eq. (7).

\section{Step V: optimization of CM interval}

Evaluate optimum CM interval to maximize the system availability by Genetic Algorithm approach (Refer Sect. 4).

The methodology suggested above is illustrated in the next section. 
Fig. 2 SMP model; CBMCentrifugal pump

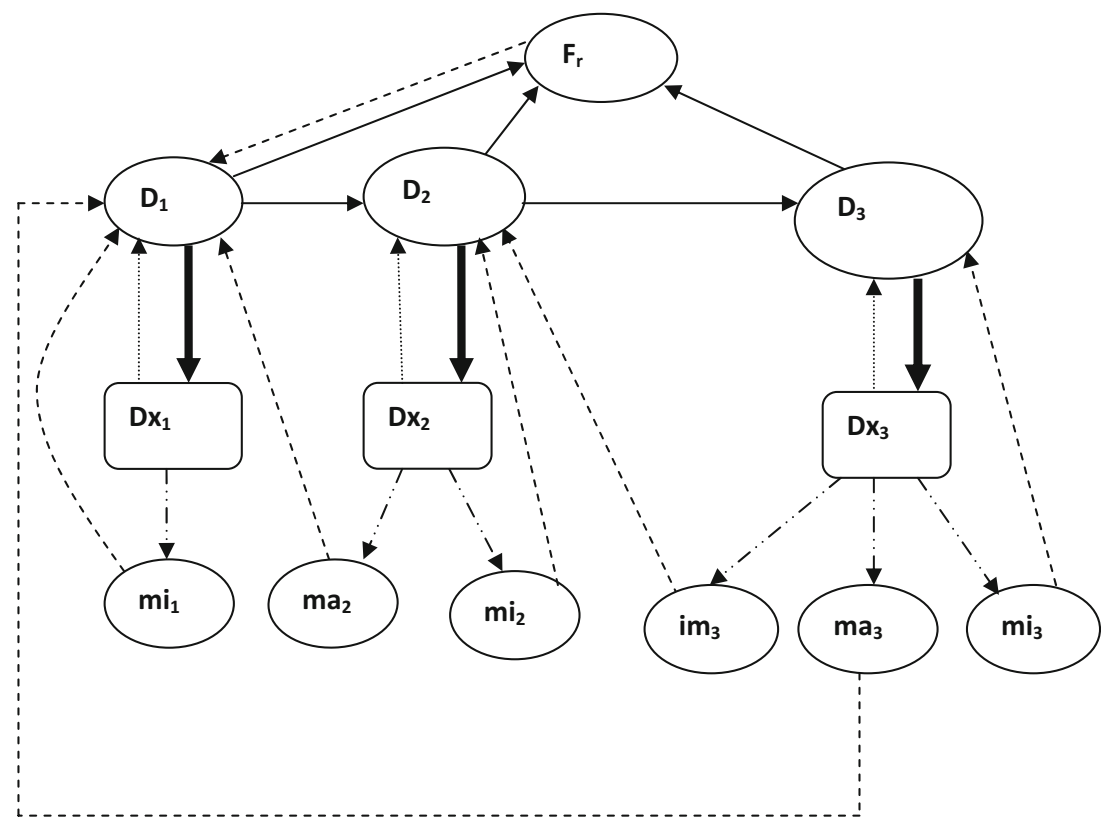

Table 1 Distribution and parameter values-Centrifugal pump

\begin{tabular}{|c|c|c|c|c|c|}
\hline State transition & Distribution & Parameter value & State transition & Distribution & Parameter value \\
\hline$D_{1} \rightarrow D_{2}$ & Weibull & $\begin{array}{l}\beta_{D_{1} D_{2}}=1.1 \\
\theta_{D_{1} D_{2}}=15000\end{array}$ & $D_{x 2} \rightarrow m i_{2}$ & Exponential & $p_{m i_{2}}=0.2, \lambda_{D_{x_{2}}}=\frac{1}{2}$ \\
\hline$D_{1} \rightarrow F_{r}$ & Exponential & $\lambda_{D_{1} F_{r}}=\frac{1}{30000}$ & $D_{x 2} \rightarrow m a_{2}$ & Exponential & $\begin{array}{l}p_{m a_{2}}=1-\left\{p_{D_{2}}+p_{m i_{2}}\right\}=0.1 \\
\lambda_{D_{x_{2}}}=\frac{1}{2}\end{array}$ \\
\hline$D_{1} \rightarrow X_{1}$ & Deterministic & $T_{1}=240$ & $D_{x 3} \rightarrow D_{3}$ & Exponential & $p_{D_{3}}=0.4, \lambda_{D_{x_{3}}}=\frac{1}{3}$ \\
\hline$D_{2} \rightarrow D_{3}$ & Weibull & $\begin{array}{l}\beta_{D_{2} D_{3}}=1.5 \\
\theta_{D_{2} D_{3}}=11000\end{array}$ & $D_{x 3} \rightarrow m i_{3}$ & Exponential & $p_{m i_{3}}=0.1, \lambda_{D_{x_{3}}}=\frac{1}{3}$ \\
\hline$D_{2} \rightarrow F_{r}$ & Exponential & $\lambda_{D_{2} F_{r}}=\frac{1}{25000}$ & $D_{x 3} \rightarrow i m_{3}^{1}$ & Exponential & $p_{i m_{3}^{1}}=0.2, \lambda_{D_{x_{3}}}=\frac{1}{3}$ \\
\hline$D_{2} \rightarrow D_{X 2}$ & Deterministic & $T_{2}=168$ & $D_{x 3} \rightarrow m a_{3}$ & Exponential & $\begin{array}{r}p_{m a_{3}}=1-\left\{p_{D_{3}}+p_{m i_{3}}+p_{i m_{3}^{1}}\right\} \\
=0.3, \lambda_{D_{x_{3}}}=\frac{1}{3}\end{array}$ \\
\hline$D_{3} \rightarrow F_{r}$ & Exponential & $\lambda_{D_{3} F_{r}}=\frac{1}{20000}$ & $m i_{1} \rightarrow D_{1}$ & Log-normal & $\mu_{m i_{1} D_{1}}=1.4, \sigma_{m i_{1} D_{1}}=0.41$ \\
\hline$D_{3} \rightarrow D_{X 3}$ & Deterministic & $T_{3}=96$ & $m i_{2} \rightarrow D_{2}$ & Log-normal & $\mu_{m i_{2} D_{2}}=1.79, \sigma_{m i_{2} D_{2}}=0.53$ \\
\hline$F_{r} \rightarrow D_{1}$ & Log-normal & $\begin{aligned} \mu_{F_{r} D_{1}} & =1.95 \\
\sigma_{F_{r} D_{1}} & =0.56\end{aligned}$ & $m i_{3} \rightarrow D_{3}$ & Log-normal & $\mu_{m i_{3} D_{3}}=2.29, \sigma_{m i_{3} D_{3}}=0.62$ \\
\hline$D_{x 1} \rightarrow D_{1}$ & Exponential & $p_{D_{1}}=0.9, \lambda_{D_{x_{1}}}=1$ & $\operatorname{im}_{3}^{1} \rightarrow D_{2}$ & Log-normal & $\mu_{i m_{3}^{1} D_{2}}=2.30, \sigma_{i m_{3}^{1} D_{2}}=0.64$ \\
\hline$D_{x 1} \rightarrow m i_{1}$ & Exponential & $\begin{array}{r}p_{m i_{1}}=1-p_{D_{1}}=0.1 \\
\lambda_{D_{x_{1}}}=1\end{array}$ & $m a_{2} \rightarrow D_{1}$ & Log-normal & $\mu_{m a_{2} D_{1}}=2.08, \sigma_{m a_{2} D_{1}}=0.59$ \\
\hline$D_{x 2} \rightarrow D_{2}$ & Exponential & $p_{D_{2}}=0.7, \lambda_{D_{x_{2}}}=\frac{1}{2}$ & $m a_{3} \rightarrow D_{1}$ & Log-normal & $\mu_{m a_{3} D_{1}}=2.48, \sigma_{m a_{3} D_{1}}=0.69$ \\
\hline
\end{tabular}

\section{Example}

A centrifugal pump is selected for steady state availability assessment using the proposed methodology involving CBM model. Condition monitoring is applied for degradation/failure detection of the pump so that a maintenance action is taken well in time to avoid the failure of the pump and increase its availability. The suggested approach is demonstrated by following its step-by-step methodology given in Sect. 5. 


\section{Step I: system description}

Pump system is considered. The centrifugal pump consists of six components, i.e., impeller, shaft, bearing, motor, bush, housing. To avoid the state space explosion problem, the states are defined at system level, i.e., at the pump level. The Semi-Markov model is developed for the system, i.e., the pump in the following step.

\section{Step II: SMP model of the pump}

Round the clock or continued operation of the pump can lead to its gradual deterioration. For the availability modeling, three degraded states are considered. Let these are: $D_{1}, D_{2}$ and $D_{3}$. A random failure state is designated as $F_{r}$. It is assumed that $\mathrm{CM}$ is performed at scheduled periodic intervals of $T_{1}$ for state $D_{1}, T_{2}$ for state $D_{2}$ and $T_{3}$ for state $D_{3}$. Let the corresponding CM metric for these degraded states are $D_{x_{1}}, D_{x_{2}}$, and $D_{x_{3}}$. The maintenance activities are performed depending upon the condition of the pump. The minor repair is considered at each stage of degradation, with three minor repair states being $m i_{1}, m i_{2}$, and $m i_{3}$.. Major repair states, $m a_{2}$ and $m a_{3}$, are considered at the second and third stage of degradation. An imperfect repair state $i m_{3}^{1}$ is envisaged at stage 3 of degradation. This is as per the discussion in Sect. 2.2.

Based on the above and referring Sect. 5.3, the SMP model is developed and is shown in Fig. 2. The state space for the model is given as:
$\Omega=\left\{D_{1}, D_{2}, D_{3} ; F_{r} ; D_{x_{1}}, D_{x_{2}}, D_{x_{3}} ; m i_{1}, m i_{2}, m i_{3} ; i m_{3}^{1} ; m a_{2}, m a_{3}\right\}$

The steady state analytical solution of the SMP model requires the state transition time distributions and their parameters, which are identified in the next step.

\section{Step III: state transition time distribution and parameters of the centrifugal pump}

The state transition time distribution and parameters for the centrifugal pump are chosen based on literature and also the experience (Nel and Haarhoff 2011; Dennis 2003). The distribution and its parameter(s) for the time duration in each state are identified and are given in Table 1.

\section{Step IV: two-stage method for steady state analysis of Centrifugal pump}

There are two stages, i.e., stage 1 and stage 2 . The stage 1 of the two-stage method is as below:

(a) Kernel matrix, $K(t)$

The Semi-Markov model is expressed by its Kernel matrix, $K(t)$ and their elements are calculated as per the method given in Sect. 3.1.1. The Kernel matrix $K(t)$ for the pump is obtained and is given as the matrix, Eq. (8), shows its zero and other leftover non-zero elements, i.e., $k_{i j}(t)$.

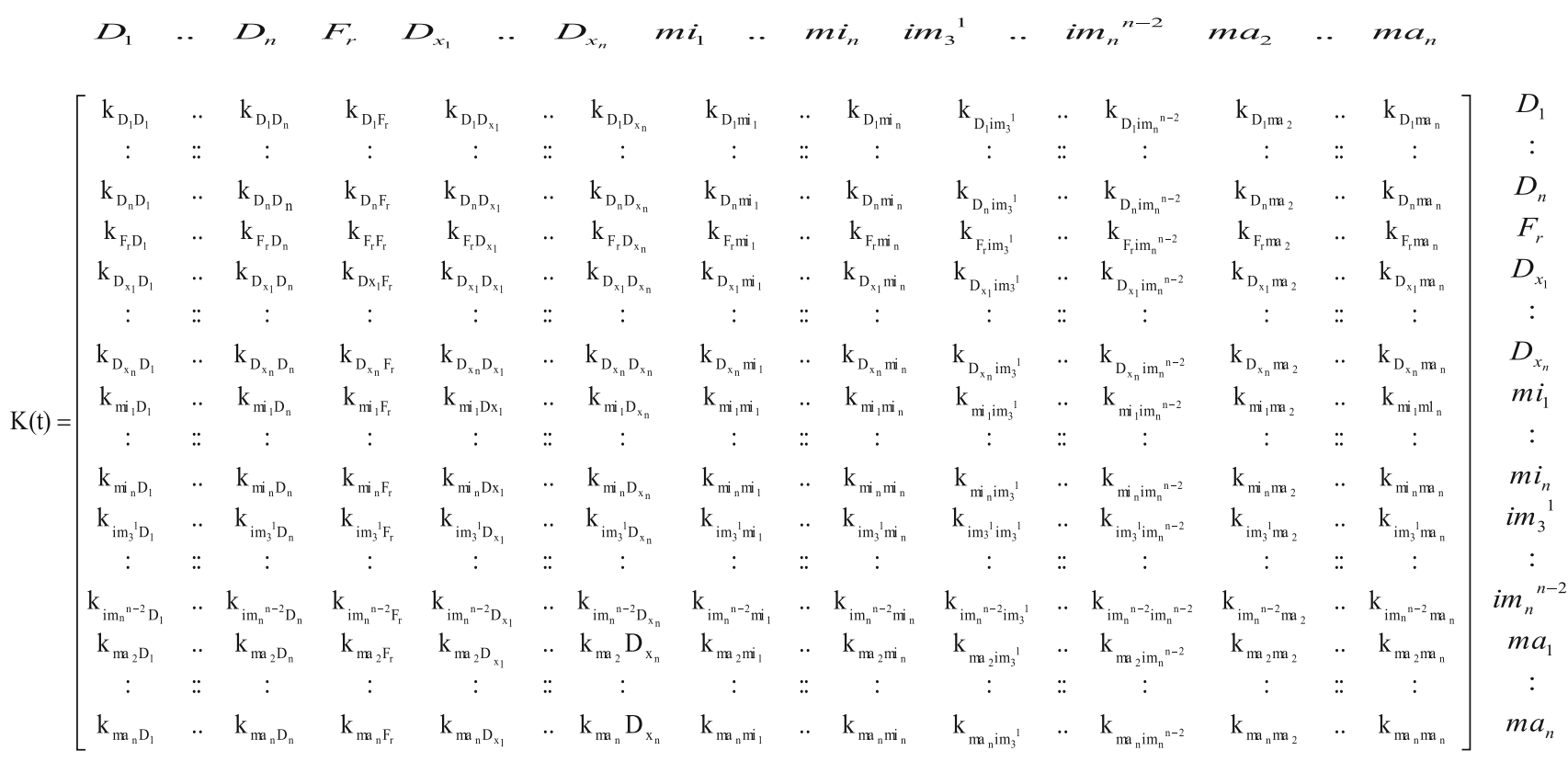


Table 2 Steady state probability of the states of the EMC-CBM model

\begin{tabular}{llll}
\hline$h_{i}$ & Probability values & $h_{i}$ & Probability values \\
\hline$h_{D_{1}}$ & 0.4579649417 & $h_{m i_{1}}$ & 0.0449538387 \\
$h_{D_{2}}$ & 0.0156699944 & $h_{m i_{2}}$ & 0.0077679296 \\
$h_{D_{3}}$ & 0.0000583264 & $h_{m i_{3}}$ & 0.0000087070 \\
$h_{F_{r}}$ & 0.0037367736 & $h_{i m_{3}^{1}}$ & 0.000174141 \\
$h_{D_{x_{1}}}$ & 0.4495383868 & $h_{m a_{2}}$ & 0.0046607578 \\
$h_{D_{x_{2}}}$ & 0.0155358593 & $h_{m a_{3}}$ & 0.0000290235 \\
$h_{D_{x_{3}}}$ & 0.0000580470 & & \\
\hline
\end{tabular}

The non-zero elements of the matrix, $K(t)$, Eq. (8), are derived, using the distributions (Table 1) and Eq. (2), and these are listed in "Appendix 1".

(b) One step transition probability matrix, $Z$, of the EMC

The Kernel matrix $K(t)$, Eq. (8), evaluates one-step transition probability matrix, $Z=K(\infty)$, of the EMC of the SMP, with $t \rightarrow \infty$. Refer Sect. 3.1.1. The elements of matrix $Z$ are evaluated with the condition that for each row, the summation of elements of $K(t)$ is 1 .

Using expressions of elements of the Kernel matrix, $K(t)$, listed in "Appendix 1", the elements of the matrix, $Z$ are derived and these are tabulated in "Appendix 2".

It may be mentioned that the Matlab software is employed to evaluate the expressions (Matlab Release 7.7 2010). The evaluated non-zero elements of the matrix, $Z$, are as listed in "Appendix 2".

(c) Steady state probability of the states of the EMC

The steady state probabilities of the EMC are evaluated using a set of Eq. (4) for the system data (Table 1).

For the model, the system of equations is:
Table 3 Steady state probability-CBM model

\begin{tabular}{llll}
\hline$P_{i}$ & Probability values & $P_{i}$ & Probability values \\
\hline$P_{D_{1}}$ & 0.9694212251 & $P_{m i_{1}}$ & 0.0017644151 \\
$P_{D_{2}}$ & 0.0233338358 & $P_{m i_{2}}$ & 0.0004763431 \\
$P_{D_{3}}$ & 0.0000497153 & $P_{m i_{3}}$ & 0.0000009276 \\
$P_{F_{r}}$ & 0.0002733783 & $P_{i m_{3}^{1}}$ & 0.0000018970 \\
$P_{D_{x_{1}}}$ & 0.0040009413 & $P_{m a_{2}}$ & 0.0003953164 \\
$P_{D_{x_{2}}}$ & 0.0002765417 & $P_{m a_{3}}$ & 0.0000039134 \\
$P_{D_{x_{3}}}$ & 0.0000015499 & & \\
\hline
\end{tabular}

expressions are listed in "Appendix 3". Using the set of expressions ("Appendix 3") and the distribution and its parameter values, given in Table 1, the sojourn time for all states, involving complex integrals, is evaluated (Matlab Release 7.7 2010) and the same are listed in "Appendix 3".

(e) Steady state probability of states of the centrifugal pump

The steady state probability of state $i$, for the pump system, is evaluated using Eq. (6), and the values of steady state probabilities of EMC, $h_{i}$, and the values sojourn times $\left(\tau_{i}\right)$ as calculated above in step IV (c) and IV (d). The evaluated value of the steady state probability of states is given in Table 3.

(f) System steady state availability for the centrifugal pump

System steady state availability assessment of the pump system is obtained using Eq. (7), i.e.,

$$
\begin{aligned}
& {\left[\begin{array}{lllllllllllll}
h_{D_{1}} & h_{D_{2}} & h_{D_{3}} & h_{F_{r}} & h_{D_{x_{1}}} & h_{D_{x_{2}}} & h_{D_{x_{3}}} & h_{m i_{1}} & h_{m i_{2}} & h_{m i_{3}} & h_{i m m_{3}^{1}} & h_{m a_{2}} & h_{m a_{3}}
\end{array}\right]=} \\
& {\left[\begin{array}{lllllllllllll}
h_{D_{1}} & h_{D_{2}} & h_{D_{3}} & h_{F_{r}} & h_{D_{x_{1}}} & h_{D_{x_{2}}} & h_{D_{x_{3}}} & h_{m i_{1}} & h_{m i_{2}} & h_{m i_{3}} & h_{i m \frac{1}{1}} & h_{m a_{2}} & h_{m a_{3}}
\end{array}\right] * Z}
\end{aligned}
$$

This set of equations is solved using Matlab (Matlab Release 7.7 2010) and the values of $h_{i}, i \in \Omega$ are tabulated and given in Table 2.

(d) Mean sojourn time of the states of the centrifugal
pump

Sojourn time values are evaluated for the stage 2 and these values are used for evaluation of steady state probabilities of system states. Sojourn time, $\tau_{i}$, expressions are derived using Eq. (5) and using the distribution parameter value given in Table 1 . These

$$
A_{\text {pump }}=\sum_{j \in W} P_{j}
$$

The set of working states of the system model (Fig. 2) is; $W=\left\{D_{1}, D_{2}, D_{3}, D_{x_{1}}, D_{x_{2}}, D_{x_{3}}\right\}$. Therefore, the availability is the sum of steady state probability of its working states, i.e.,

$A_{\text {pump }}=P_{D_{1}}+P_{D_{2}}+P_{D_{3}}+P_{D_{x_{1}}}+P_{D_{x_{2}}}+P_{D_{x_{3}}}$

The evaluated value of steady state availability is:

$A_{\text {pump }}=0.9971375783$ 
The result obtained from the proposed model is in close agreement with the result (0.99970) following the same input data with different approach (Nel and Haarhoff 2011).

\section{Step-V: optimization of CM interval using GA}

The optimum CM interval to maximize the system availability is obtained by Genetic Algorithm approach-refer Sect. 4 for details. A code for availability following the above steps is written in Matlab and the availability function is called in the GA tool box of Matlab to find the optimum values of three CM intervals; $\mathrm{CM}$ intervals; $\mathrm{T}_{1}, \mathrm{~T}_{2}$ and $\mathrm{T}_{3}$, and system availability, A. The results obtained are: $T_{1}=9650 \mathrm{~h} ; T_{2}=9600 \mathrm{~h}$; $T_{3}=9520 \mathrm{~h}$ and $A=0.999446816$.

The results obtained are realistic and attributed to the SMP model, incorporating multi-state degradation, appropriate distribution for modeling random failures, repairs and $\mathrm{CM}$ interval. The suggested methodology is useful for maintenance engineers in selecting suitable maintenance policies in the plant to achieve the system availability goals. Also, designers can use the methodology to design mechanical systems for desired availability by introducing the appropriate repair and level of repair.

\section{Conclusion}

In this paper, condition-based maintenance model of mechanical systems are proposed for steady-state availability analysis using the SMP. The system model is developed considering multi-state degradation, using condition-based maintenance. In addition to degradation, random failures are also considered. Weibull, log-normal, exponential and deterministic distributions are considered for degradation, repair, random failure and periodic CM, respectively. It is demonstrated through a case study that condition-monitoring can significantly improve the system availability. Also, the optimum CM interval is determined for maximizing the system availability using Genetic Algorithm approach.

The proposed methodology is quicker than the simulation-based approaches since results are obtained in a single run. Moreover, the results are more accurate than the results obtained from simulation approach as these are obtained from closed form solutions. Since appropriate distributions for the failure and the repair time are used, the results obtained with suggested methodology are more realistic. The model can be manipulated under different set of parameter values to see the impact of various maintenance policies on system availability. The proposed methodology is useful for maintenance engineers in decision making, and directly helps them for devising suitable maintenance/replacement policies in the plant.

Open Access This article is distributed under the terms of the Creative Commons Attribution 4.0 International License (http://crea tivecommons.org/licenses/by/4.0/), which permits unrestricted use, distribution, and reproduction in any medium, provided you give appropriate credit to the original author(s) and the source, provide a link to the Creative Commons license, and indicate if changes were made.

\section{Appendix 1}

See Table 4.
Table 4 Elements (non-zero) of global kernel matrix, $K(t)-\mathrm{CBM}$ model

\begin{tabular}{|c|c|c|}
\hline$k_{i j}$ & $k_{i j}(t)$ & $k_{i j}(t)$ with specific distribution \\
\hline$k_{D_{1} D_{2}}$ & $\int_{0}^{t} \bar{F}_{D_{1} D_{x_{1}}} \bar{F}_{D_{1} F_{r}} d F_{D_{1} D_{2}}$ & $\frac{\beta_{D_{1} D_{2}}}{\theta_{D_{1} D_{2}}^{\beta_{1} D_{2}}} \int_{0}^{t} U\left(x-T_{1}\right) x^{\beta_{D_{1} D_{2}}-1} e^{-\left[\left(\frac{x}{\theta_{D_{1} D_{2}}}\right)^{\beta_{D_{1}} D_{2}}+\lambda_{D_{1} F_{r}} x\right]} d x$ \\
\hline$k_{D_{1} F_{r}}$ & $\int_{0}^{t} \bar{F}_{D_{1} D_{x_{1}}} \bar{F}_{D_{1} D_{2}} d F_{D_{1} F_{r}}$ & $\lambda_{D_{1} F_{r}} \int_{0}^{t} U\left(x-T_{1}\right) e^{-\left[\left(\frac{x}{\sigma_{D_{1} D_{2}}}\right)^{\beta_{D_{1}} D_{2}}+\lambda_{D_{1} F_{r}} x\right]} d x$ \\
\hline$k_{D_{1} D_{x_{1}}}$ & $\int_{0}^{t} \bar{F}_{D_{1} F_{r}} \bar{F}_{D_{1} D_{2}} d F_{D_{1} D_{x_{1}}}$ & $\int_{0}^{t} e^{-\left[\left(\frac{x}{\bar{\theta}_{D_{1} D_{2}}}\right)^{\left.\beta_{D_{1} D_{2}}+\lambda_{D_{1} F_{r}} x\right]}\right.} d\left(U\left(x-T_{1}\right)\right)$ \\
\hline$k_{D_{2} D_{3}}$ & $\int_{0}^{t} \bar{F}_{D_{2} D_{x_{2}}} \bar{F}_{D_{2} F_{r}} d F_{D_{2} D_{3}}$ & $\frac{\beta_{D_{2} D_{3}}}{\theta_{D_{2} D_{3}}^{\beta_{2} D_{3}}} \int_{0}^{t} U\left(x-T_{2}\right) x^{\beta_{D_{2} D_{3}}-1} e^{-\left[\left(\frac{x}{\theta_{D_{2} D_{3}}}\right)^{\beta_{D_{2} D_{3}}}+\lambda_{D_{2} F_{r}} x\right]} d x$ \\
\hline$k_{D_{2} F_{r}}$ & $\int_{0}^{t} \bar{F}_{D_{2} D_{x_{2}}} \bar{F}_{D_{2} D_{3}} d F_{D_{2} F_{r}}$ & $\lambda_{D_{2} F_{r}} \int_{0}^{t} U\left(x-T_{2}\right) e^{-\left[\left(\frac{x}{\theta_{D_{2}} D_{3}}\right)^{\beta_{D_{2}} D_{3}}+\lambda_{D_{2} F_{r}} x\right]} d x$ \\
\hline$k_{D_{2} D_{x_{2}}}$ & $\int_{0}^{t} \bar{F}_{D_{2} F_{r}} \bar{F}_{D_{2} D_{3}} d F_{D_{2} D_{x_{2}}}$ & $\int_{0}^{t} e^{-\left[\left(\frac{x}{{ }_{D_{2}} D_{3}}\right)^{\beta_{D_{2} D_{3}}}+\lambda_{D_{2} F_{r}} x\right]} d\left(U\left(x-T_{2}\right)\right)$ \\
\hline$k_{D_{3} F_{r}}$ & $\int_{0}^{t} \bar{F}_{D_{3} D_{x_{3}}} d F_{D_{3} F_{r}}$ & $\lambda_{D_{3} F_{r}} \int_{0}^{t} U\left(x-T_{3}\right) e^{-\lambda_{D_{3} F_{r}} x} d x$ \\
\hline$k_{D_{3} D_{x_{3}}}$ & $\int_{0}^{t} \bar{F}_{D_{3} F_{r}} d F_{D_{3} D_{x_{3}}}$ & $\int_{0}^{t} e^{-\lambda_{D_{3} F_{r}} x} d\left(U\left(x-T_{3}\right)\right)$ \\
\hline
\end{tabular}


Table 4 continued

\begin{tabular}{|c|c|c|}
\hline$k_{i j}$ & $k_{i j}(t)$ & $k_{i j}(t)$ with specific distribution \\
\hline$k_{F_{r} D_{1}}$ & $F_{F_{r} D_{1}}(t)$ & $\frac{1}{2}+\frac{1}{2} \operatorname{erf}\left(\frac{\ln (x)-\mu_{F_{r} D_{1}}}{\sqrt{2} \sigma_{F_{r} D_{1}}}\right)$ \\
\hline$k_{D_{x x} D_{1}}$ & $p_{D_{1}} F_{D_{x_{1}}}$ & $p_{D_{1}}\left(1-e^{-\lambda_{D_{x_{1}}} t}\right)$ \\
\hline$k_{D_{x_{1}} m i_{1}}$ & $\left(1-p_{D_{1}}\right) F_{D_{x_{1}}}$ & $\left(1-p_{D_{1}}\right)\left(1-e^{-\lambda_{D_{x_{1}}} t}\right)$ \\
\hline$k_{D_{x_{2}} D_{2}}$ & $p_{D_{2}} F_{D_{x_{2}}}$ & $p_{D_{2}}\left(1-e^{-\lambda_{D_{X_{2}}} t}\right)$ \\
\hline$k_{D_{x_{2}} m i_{2}}$ & $p_{m i_{2}} F_{D_{x_{2}}}$ & $p_{m i_{2}}\left(1-e^{-\lambda_{D_{x_{2}}} t}\right)$ \\
\hline$k_{D_{x_{2}} m a_{2}}$ & $\begin{array}{c}\left\{1-\left(p_{D_{2}}+\right.\right. \\
\left.\left.p_{m i_{2}}\right)\right\} F_{D_{x_{2}}}\end{array}$ & $\begin{array}{r}\left\{1-\left(p_{D_{2}}+p_{m i_{2}}\right)\right\} \\
\left(1-e^{-\lambda_{D_{x_{2}}} t}\right)\end{array}$ \\
\hline$k_{D_{x_{3}} D_{3}}$ & $p_{D_{3}} F_{D_{x_{3}}}$ & $p_{D_{3}}\left(1-e^{-\lambda_{D_{x_{3}}} t}\right)$ \\
\hline$k_{D_{x_{3}} m i_{3}}$ & $p_{m i_{3}} F_{D_{x_{3}}}$ & $p_{m i_{3}}\left(1-e^{-\lambda_{D_{x_{3}}} t}\right)$ \\
\hline$k_{D_{x_{3}} i m_{3}^{1}}$ & $p_{i m_{3}^{1}} F_{D_{x_{3}}}$ & $p_{i m_{3}^{1}}\left(1-e^{-\lambda_{D_{x_{3}}} t}\right)$ \\
\hline$k_{D_{x_{3}} m a_{3}}$ & $\begin{array}{r}\left\{1-\left(p_{D_{3}}+p_{m i_{3}}\right.\right. \\
\left.\left.+p_{i m_{3}^{1}}\right) F_{D_{x_{3}}}\right\}\end{array}$ & $\left\{1-\left(p_{D_{3}}+p_{m i_{3}}+p_{i m_{3}^{1}}\right)\right\}\left(1-e^{-\lambda_{D_{x_{3}}} t}\right)$ \\
\hline$k_{m i_{1} D_{1}}$ & $F_{m i_{1} D_{1}}(t)$ & $\frac{1}{2}+\frac{1}{2} \operatorname{erf}\left(\frac{\ln (x)-\mu_{m i_{1} D_{1}}}{\sqrt{2} \sigma_{m i_{1} D_{1}}}\right)$ \\
\hline$k_{m i_{2} D_{2}}$ & $F_{m i_{2} D_{2}}(t)$ & $\frac{1}{2}+\frac{1}{2} \operatorname{erf}\left(\frac{\ln (x)-\mu_{m i_{2} D_{2}}}{\sqrt{2} \sigma_{m i_{2} D_{2}}}\right)$ \\
\hline$k_{m i_{3} D_{3}}$ & $F_{m i_{3} D_{3}}(t)$ & $\frac{1}{2}+\frac{1}{2} \operatorname{erf}\left(\frac{\ln (x)-\mu_{m i_{3} D_{3}}}{\sqrt{2} \sigma_{m i_{3} D_{3}}}\right)$ \\
\hline$k_{i m_{3}^{1} D_{2}}$ & $F_{i m_{3}^{1} D_{2}}(t)$ & $\frac{1}{2}+\frac{1}{2} \operatorname{erf}\left(\frac{\ln (x)-\mu_{m i i_{3}^{1} D_{2}}}{\sqrt{2} \sigma_{m i i_{3}^{3} D_{2}}}\right)$ \\
\hline$k_{m a_{2} D_{1}}$ & $F_{m a_{2} D_{1}}(t)$ & $\frac{1}{2}+\frac{1}{2} \operatorname{erf}\left(\frac{\ln (x)-\mu_{m a_{2} D_{1}}}{\sqrt{2} \sigma_{m a_{2} D_{1}}}\right)$ \\
\hline$k_{m a_{3} D_{1}}$ & $F_{m a_{3} D_{1}}(t)$ & $\frac{1}{2}+\frac{1}{2} \operatorname{erf}\left(\frac{\ln (x)-\mu_{m a_{3} D_{1}}}{\sqrt{2} \sigma_{m a_{3} D_{1}}}\right)$ \\
\hline
\end{tabular}

\section{Appendix 2}

\section{See Table 5.}

Table 5 Non-zero elements of one step transition probability matrix, $Z-\mathrm{CBM}$ model

\begin{tabular}{|c|c|c|}
\hline$p_{i j}$ & Expressions of Elements of matrix, $Z, p_{i j}=k_{i j}(\infty)$ & $p_{i j}$ values \\
\hline$p_{D_{1} D_{2}}$ & $\frac{\beta_{D_{1} D_{2}}}{\theta_{D_{1} D_{2}}^{\beta_{1} D_{2}}} \int_{0}^{T_{1}} x^{\beta_{D_{1} D_{2}}-1} e^{-\left[\left(\frac{x}{\theta_{D_{1} D_{2}}}\right)^{\beta_{D_{1}} D_{2}}+\lambda_{D_{1} F_{r}} x\right]} d x$ & 0.01047 \\
\hline$p_{D_{1} F_{r}}$ & $\lambda_{D_{1} F_{r}} \int_{0}^{T_{1}} e^{-\left[\left(\frac{x}{\partial_{D_{1} D_{2}}}\right)^{\beta_{D_{1}} D_{2}}+\lambda_{D_{1} F_{r}} x\right]} d x$ & 0.00793 \\
\hline$p_{D_{1} D_{x_{1}}}$ & $1-\left\{k_{D_{1} D_{2}}(\infty)+k_{D_{1} F_{r}}(\infty)\right\}$ & 0.98160 \\
\hline$p_{D_{2} D_{3}}$ & $\frac{\beta_{D_{2} D_{3}}}{\theta_{D_{2} D_{3}}^{\beta_{2} D_{3}}} \int_{0}^{T_{2}} x^{\beta_{D_{2} D_{3}}-1} e^{-\left[\left(\frac{x}{\theta_{D_{2} D_{3}}}\right)^{\beta_{D_{2}} D_{3}}+\lambda_{D_{2} F_{r}} x\right]} d x$ & 0.00187 \\
\hline$p_{D_{2} F_{r}}$ & $\lambda_{D_{2} F_{r}} \int_{0}^{T_{2}} e^{-\left[\left(\frac{x}{\sigma_{D_{2}} D_{3}}\right)^{\left.\beta_{D_{2} D_{3}}+\lambda_{D_{2} F_{r}} x\right]}\right.} d x$ & 0.00669 \\
\hline$p_{D_{2} D_{x_{2}}}$ & $1-\left\{k_{D_{2} D_{3}}(\infty)+k_{D_{2} F_{r}}(\infty)\right\}$ & 0.99144 \\
\hline$p_{D_{3} F_{r}}$ & $\lambda_{D_{3} F_{r}} \int_{0}^{T_{3}} e^{-\lambda_{D_{3} F_{r}} x} d x$ & 0.00479 \\
\hline$p_{D_{3} D_{x_{3}}}$ & $1-k_{D_{3} F_{r}}(\infty)$ & 0.99521 \\
\hline
\end{tabular}


Table 5 continued

\begin{tabular}{|c|c|c|c|c|c|}
\hline$p_{i j}$ & \multicolumn{4}{|c|}{ Expressions of Elements of matrix, $Z, p_{i j}=k_{i j}(\infty)$} & $p_{i j}$ values \\
\hline$p_{D_{x_{1}} D_{1}}$ & \multicolumn{3}{|c|}{$p_{D_{1}}\left(1-e^{-\lambda_{D_{x_{1}}} t}\right)$} & & 0.9 \\
\hline$p_{D_{x_{1} m i_{1}}}$ & \multicolumn{3}{|c|}{$\left(1-p_{D_{1}}\right)\left(1-e^{-\lambda_{D_{x_{1}}} t}\right)$} & & 0.1 \\
\hline$p_{D_{x_{2}} m a_{2}}$ & \multicolumn{3}{|c|}{$1-\left(p_{D_{2}}+p_{m i_{2}}\right)$} & & 0.3 \\
\hline$p_{D_{x_{3}} m a_{3}}$ & \multicolumn{3}{|c|}{$1-\left(p_{D_{3}}+p_{m i_{3}}+p_{i m_{3}^{1}}\right)$} & & 0.5 \\
\hline$p_{i j}$ & $\begin{array}{l}\text { Expressions of Elements of } \\
\text { matrix, } Z, p_{i j}=k_{i j}(\infty)\end{array}$ & $\begin{array}{l}p_{i j} \\
\text { values }\end{array}$ & $p_{i j}$ & $\begin{array}{l}\text { Expressions of Elements of } \\
\text { matrix, } Z, p_{i j}=k_{i j}(\infty)\end{array}$ & $\begin{array}{l}p_{i j} \\
\text { values }\end{array}$ \\
\hline$p_{F_{r} D_{1}}$ & 1 & 1 & $p_{m i_{1} D_{1}}$ & 1 & 1 \\
\hline$p_{D_{x_{2}} D_{2}}$ & $p_{D_{2}}$ & 0.2 & $p_{m i_{2} D_{2}}$ & 1 & 1 \\
\hline$p_{D_{x_{2}} m i_{2}}$ & $p_{m i_{2}}$ & 0.5 & $p_{m i_{3} D_{3}}$ & 1 & 1 \\
\hline$p_{D_{x_{3}} D_{3}}$ & $p_{D_{3}}$ & 0.05 & $p_{i m_{3}^{1} D_{2}}$ & 1 & 1 \\
\hline$p_{D_{x_{3}} m i_{3}}$ & $p_{m i_{3}}$ & 0.15 & $p_{m a_{2} D_{1}}$ & 1 & 1 \\
\hline$p_{D_{x_{3}} i m_{3}^{1}}$ & $p_{i m_{3}^{1}}$ & 0.3 & $p_{m a_{3} D_{1}}$ & 1 & 1 \\
\hline
\end{tabular}

\section{Appendix 3}

See Table 6.

Table 6 Mean sojourn time expressions-CBM model

\begin{tabular}{|c|c|c|c|}
\hline$\tau_{i}$ & Sojourn time expressions & Sojourn time expressions with parameter values & Sojourn time values \\
\hline$\tau_{D_{1}}$ & $\int_{0}^{\infty} \bar{F}_{D_{1} D_{x_{1}}} \bar{F}_{D_{1} F_{r}} \bar{F}_{D_{1} D_{2}} d x$ & $\int_{0}^{T_{2}} e^{-\left[\left(\frac{x}{\theta_{D_{2}} D_{3}}\right)^{\beta_{D_{2}} D_{3}}+\lambda_{D_{2} F_{r}} x\right]} \mathrm{d} x$ & 237.84 \\
\hline$\tau_{D_{2}}$ & $\int_{0}^{\infty} \bar{F}_{D_{2} D_{x_{2}}} \bar{F}_{D_{2} F_{r}} \bar{F}_{D_{2} D_{3}} d x$ & $\int_{0}^{T_{2}} e^{-\left[\left(\frac{x}{\theta_{D_{2} D_{3}}}\right)^{\left.\beta_{D_{2} D_{3}}+\lambda_{D_{2} F_{r}} x\right]}\right.} \mathrm{d} x$ & 167.31 \\
\hline$\tau_{D_{3}}$ & $\int_{0}^{\infty} \bar{F}_{D_{3} D_{x_{3}}} \bar{F}_{D_{3} F_{r}} d x$ & $\int_{0}^{T_{3}} e^{-\lambda_{D_{3} F_{r}} x} d x$ & 95.77 \\
\hline$\tau_{F_{r}}$ & $\int_{0}^{\infty} \bar{F}_{F_{r} D_{1}} d x$ & $e^{\mu_{F_{r} D_{1}}+\frac{1}{2} \sigma_{F_{r} D_{1}}^{2}}$ & 8.22 \\
\hline$\tau_{D_{x_{1}}}$ & $\int_{0}^{\infty} \bar{F}_{D_{x_{1}}} d x$ & $\frac{1}{\lambda_{D_{x_{1}}}}$ & 1 \\
\hline$\tau_{D_{x_{2}}}$ & $\int_{0}^{\infty} \bar{F}_{D_{x_{2}}} d x$ & $\frac{1}{\lambda_{D_{x_{2}}}}$ & 2 \\
\hline$\tau_{D_{x_{3}}}$ & $\int_{0}^{\infty} \bar{F}_{D_{x_{3}}} d x$ & $\frac{1}{\lambda_{D_{x_{3}}}}$ & 3 \\
\hline$\tau_{m i_{1}}$ & $\int_{0}^{\infty} \bar{F}_{m i_{1} D_{1}} d x$ & $e^{\mu_{m i_{1} D_{1}}+\frac{1}{2} \sigma_{m i_{1} D_{1}}^{2}}$ & 4.41 \\
\hline$\tau_{m i_{2}}$ & $\int_{0}^{\infty} \bar{F}_{m i_{2} D_{2}} d x$ & $e^{\mu_{m i_{2} D_{2}}+\frac{1}{2} \sigma_{m i_{2} D_{2}}^{2}}$ & 6.89 \\
\hline$\tau_{m i_{3}}$ & $\int_{0}^{\infty} \bar{F}_{m i_{3} D_{3}} d x$ & $e^{\mu_{m i_{3} D_{3}}+\frac{1}{2} \sigma_{m i_{3} D_{3}}^{2}}$ & 11.97 \\
\hline$\tau_{i m_{3}^{1}}$ & $\int_{0}^{\infty} \bar{F}_{i m_{3}^{1} D_{2}} d x$ & $e^{\mu_{i m_{3}^{1} D_{2}}+\frac{1}{2} \sigma_{i m_{3}^{1} D_{2}}^{2}}$ & 12.24 \\
\hline$\tau_{m a_{2}}$ & $\int_{0}^{\infty} \bar{F}_{m a_{2} D_{1}} d x$ & $e^{\mu_{m a_{2} D_{1}}+\frac{1}{2} \sigma_{m a_{2} D_{1}}^{2}}$ & 9.53 \\
\hline$\tau_{m a_{3}}$ & $\int_{0}^{\infty} \bar{F}_{m a_{3} D_{1}} d x$ & $e^{\mu_{m a_{3} D_{1}}+\frac{1}{2} \sigma_{m a_{3} D_{1}}^{2}}$ & 15.15 \\
\hline
\end{tabular}




\section{References}

Ahmad R, Kamaruddin S, Azid I, Almanar I (2011) Maintenance management decision model for preventive maintenance strategy on production equipment. J Ind Eng Int 7(13):22-34

Ahmad A, Raissi S, Khalili-Damghani K (2017) A simulation-based optimization approach for free distributed repairable multi-state availability-redundancy allocation problems. Reliab Eng Syst Saf 157:177-191

Amari SV, McLaughlin L (2004) Optimal design of a condition-based maintenance model. In: Proceedings of IEEE annual reliability and maintainability symposium, Los Angeles, USA, 26-29 January, pp 528-533

Carr MJ, Wang W (2011) An approximate algorithm for prognostic modeling using condition monitoring information. Eur $\mathrm{J}$ Oper Res 211(1):90-96

Charles-Owaba OE, Oluleye AE, Oyawale FA, Oke SA (2008) An opportunity cost maintenance scheduling framework for a fleet of ships: a case study. J Ind Eng Int 4(6):64-77

Chen D, Trivedi KS (2005) Optimization for condition-based maintenance with semi-Markov decision process. Reliab Eng Syst Saf 90(1):25-29

Crocker J, Kumar UD (2000) Age-related maintenance versus reliability centered maintenance: a case study on aero-engines. Reliab Eng Syst Saf 67(2):113-118

Dennis A (2003) Application of MCS system reliability analysis. In: Proceedings of TAMU, 20th international pump users symposium. Department of Mechanical Engineering, Turbo machinery laboratory, Texas A \& M University, College Station, Houston, Texas, USA, 17-20 March, pp 91-94

El-Damcese MA, Temraz NS (2011) Availability and reliability measures for multi-state system by using Markov reward model. Reliability Theory and Application 6(3):68-85

Fricks RM, Trivedi KS (1997) Modeling failure dependencies in reliability analysis using stochastic Petri nets. In: Proceedings of SCS Europe 11th European simulation multiconference, Istanbul, Turkey, 1-4 June, pp 1-22

Goldberg DE (2006) Genetic algorithm. Pearson Education India, New Delhi

Jager P, Bertsche B (2004) A new approach to gathering failure behaviour information about mechanical components based on expert knowledge. In: Proceedings of IEEE annual reliability and maintainability symposium, Los Angeles, USA, 26-29 January, pp 90-95

Jardine AKS, Lin D, Banjevic D (2006) A review on machinery diagnostic and prognostics implementing condition-based maintenance. Mech Syst Signal Process 20(7):1483-1510
Khanduja R, Tewari PC, Kumar D (2011) Steady state behavior and maintenance planning of bleaching system in a paper plant. J Ind Eng Int 7(12):39-44

Kulkrani VG (2009) Modeling and analysis of stochastic systems. Chapman and Hall, London

Kumar S, Tewari PC, Kumar S (2009) Performance evaluation and availability analysis of ammonia synthesis unit in a fertilizer plant. J Ind Eng Int 5(9):17-26

Kumar G, Jain V, Gandhi OP (2014) Steady-state availability analysis of repairable mechanical systems with opportunistic maintenance by using semi-Markov process. Int $\mathbf{J}$ Syst Assur Eng Manag 5(4):664-678

Kuo W, Rajendra VP, Tillman FA, Hwang CL (2001) Optimal reliability design fundamentals and applications. Cambridge University Press, London

Majid MAA, Nasir M (2011) Multi-state system availability model of electricity generation for a cogeneration district cooling plant. Asian J Appl Sci 4(4):431-438

MATLAB release 7.7 (2010) The Math Works Inc. Natick, Massachusetts

Nel DT, Haarhoff J (2011) Bulk water distribution power supply failures. J S Afr Inst Civ Eng 51(1):55-60

Pandian A, Ali A (2009) A review of recent trends in machine diagnosis and prognosis algorithm. In: Proceedings of IEEE World congress on nature \& biologically inspired computing, Coimbatore, India, 9-11 December, pp 1731-1736

Qiang H, Zhihua D, Xiao Z (2010) Application of HSMM on NC machine's state recognition. In: Proceedings of IEEE international conference on e-health networking, digital ecosystems and technologies, Shenzhen, China, 17-18 April, pp 189-191

Sim SH, Endrenyi J (1988) Optimal preventive maintenance with repair. J IEEE Trans Reliab 37(1):92-96

Van PD, Voisin A, Levrat E, Iung B (2012) Condition-based maintenance with both perfect and imperfect maintenance actions. In: Proceedings of PHM society annual conference of the prognostics and health management society, Minnesota, USA, 23-27 September, pp 3-9

Wang W (2008) Condition based maintenance modeling. In: Kobbacy KAH, Murty DNP (eds) Complex system maintenance handbook, Part C. Springer, London, pp 111-131

Wang N, Sun S, Li S, Si S (2010) Modeling and optimization of deteriorating equipment with predictive maintenance and inspection. In: Proceedings of IEEE 17th international conference on industrial engineering and engineering management, Xiamen, China, 29-31 October, pp 942-946

Xie W, Hong Y, Trivedi K (2005) Analysis of a two-level software rejuvenation policy. Reliab Eng Syst Saf 87(1):13-22 